\title{
Thanks to our reviewers
}

We would like to gratefully acknowledge all reviewers for their time and expertise so generously given to the peer review of manuscripts in the Journal of Remanufacturing in 2018. We truly appreciate their continued support and commitment in helping us maintain and improve the quality of our journal.

\author{
Kamel Agroui \\ Hasan Aksoy \\ Emel Aktas \\ Bandar Alkhayyal \\ Anna Aminoff \\ Mats Björkman \\ Chandra Garg \\ Jighyasu Gaur \\ B.C. Giri \\ Maria Grazia Gnoni \\ Paulina Golinska \\ Madhav Govind \\ Laura Hay \\ Winifred Ijomah
}

Zhigang Jiang
Ana Jimenez Rivero
Yong-Sung Jun
Chih-chuan Kao
Sachin Kumar
Jelena Kurilova-Palisaitiene
Mattias Lindahl
Xichun Luo
Mitsutaka Matsumoto
Sandeep Mondal
David Paterson
Pedro Piñeyro
Amar Ramudhin
Amir Rashid

Sascha Ruggaber

Rashed Sahraeian

Tomohiko Sakao

Chun $\mathrm{Su}$

Suresh Subramoniam

Erik Sundin

Ata Allah Taleizadeh

Ou Tang

Ming-Lang Tseng

Patricia van Loon

Haiyan Wang

James Windmill

Li Zhou 